\title{
A MYC target long non-coding RNA GATA2-AS1 regulates non-small cell lung cancer growth
}

\author{
L. ZHANG ${ }^{1, *}$, L. GAO ${ }^{2, *}$, M. SHAO ${ }^{1}$, G. Y. SUN ${ }^{3, *}$ \\ ${ }^{1}$ Department of Critical Care Medicine, The First Affiliated Hospital of Anhui Medical University, Hefei, China; ${ }^{2}$ Department of Respiratory and \\ Critical Care Medicine, The Second Hospital of Anhui Medical University, Hefei, China; ${ }^{3}$ Department of Respiratory and Critical Care Medicine, \\ The First Affiliated Hospital of Anhui Medical University, Hefei, China
}

*Correspondence: sungengy@126.com

${ }^{*}$ Contributed equally to this work.

Received February 10, 2019 / Accepted June 6, 2019

\begin{abstract}
Non-small cell lung cancer (NSCLC) is the most prevalent subtype of lung cancer histologically, and an increasing number of evidences have shown during the past years that long non-coding RNAs (lncRNAs) are involved in tumorigenesis. Here we found a long non-coding RNA, GATA2-AS1, repressing NSCLC cells proliferation via regulating GATA2. GATA2-AS1 gene is located at antisense strand of GATA2 on chromosome while GATA2-AS1 RNA interacts with GATA1 protein at promoter region of GATA2 and then inhibits its transcription. Moreover, GATA2-AS1 is transcriptionally repressed by MYC in NSCLC cells. To conclude, our study discovered the role of lncRNA GATA2-AS1 in human non-small cell lung cancer growth thus providing a potential target for lung cancer drugs.
\end{abstract}

Key words: NSCLC, IncRNA, MYC, GATA2

Lung cancer is one of the most prevalent types of cancer with poor prognosis and high mortality, and non-small cell lung cancer (NSCLC) is classified as the most common group during lung cancer diagnosis [1]. RAS pathway genes, such as GTPases and receptor tyrosine kinases (RTKs), are frequently mutated in NSCLC patients $[2,3]$. GATA2 gene is crucial for growth of those lung cancers with mutations in KRAS and other oncogenes on the RTK/RAS pathway. GATA2 deficiency reduces the viability of NSCLC cells with RAS pathway mutations and dramatically represses NSCLC development. Studies have discovered that GATA2 maintains lung cancer cells survival through concurrent modulation of three pathways: the proteasome complex, the IL-1/NF-kB signaling, and the Rho-signaling [4].

The GATA family members, such as GATA2, regulate a multitude of developmental processes as transcription factors [5]. Interactions among GATA transcription factors play an important role in control of GATA family members expression [6]. For example, GATA2 binds at GATA2 gene promoter region and activates its own transcription. However, GATA1 can displace GATA2 from this position, and instigates GATA2 repression by means of disruption of its positive autoregulation $[6,7]$. Therefore GATA1-mediated displacement of GATA2 is tightly coupled to repression of GATA2 transcription.

MYC, often constitutively expressed in many types of cancer cells, is a transcription factor that binds to enhancer box sequences (E-boxes), thus activating many pro-proliferative genes expression [8-10]. It leads to an increased level of many oncogenic genes involved in abnormal cell proliferation and then contributes to the formation of cancer [11, 12]. Constitutive upregulation of MYC gene has been discovered in a lot of carcinomas, such as the cervix, colon, breast, stomach and lung cancers. As a result, MYC is considered to be a promising target for cancer treatment [13-15].

During past years long non-coding RNAs have been found to participate in modulation of tumorigenesis $[16,17]$. LncRNAs are defined as transcripts longer than 200 nucleotides, which are not translated into protein [18]. LncRNAs can target transcriptional activators or repressors to regulate gene transcription and expression [19]. One type of lncRNAs accumulates in cis after transcription and regulate adjacent gene expression either by recruiting transcription factors or chromatin modifiers [20]. For example, lncRNAs expression can be induced at CCND1 promoter region after DNA damage, which then bind to TLS protein in cis and subse- 
quently inhibit histone acetyltransferase function and CCND1 transcription [21]. Most eukaryotic promoters are bidirectional, so that initiating Polymerase II can generate a transcript in either direction: the sense (mRNA) direction or the antisense direction. Here we report an antisense lncRNA of GATA2 gene, which is transcriptionally regulated by MYC and inhibits GATA2 expression by interacting with GATA1 protein at GATA2 promoter.

\section{Materials and methods}

Reagents and antibodies. The following antibodies used in this study were purchased from the indicated sources: rabbit IgG and anti-Flag (Sigma); anti-H2A, anti-GATA1 and anti-MYC (Cell Signaling Technology); anti-GATA2, antiFOG1anti-p65, anti-p-p65, anti-IkBa, anti-IkB $\alpha$ and anti- $\beta$ Actin (Santa Cruz). The primers and siRNAs used in this study are obtained from IDT (Integrated Device Technology) and their sequences are listed in Table S1.

Cell culture and transfection. Cell culture and transfection were performed as previously described [22]. Briefly, cells were cultured in DMEM supplemented with $10 \%$ fetal bovine serum, $1 \mathrm{mM}$ sodium pyruvate and $100 \mathrm{mg} / \mathrm{ml}$ streptomycin (Gibco).

RNA interference. HEK 293T cells were transfected with scramble or indicated siRNA (GATA2-AS1 siRNA sequence GCAGCUUACGAUUCUUCAU, Miz1 siRNA sequence was GCAAGAAAUGGAGGUGGAG) and cultured in Opti-MEM. Twelve hours later, cells were switched to DMEM culture medium with $10 \%$ FBS for an additional $48 \mathrm{~h}$. The efficiency of knockdown was assessed by western blotting and quantitative PCR (qPCR) analysis.

Quantitative RT-PCR. Total RNA was extracted using Trizol (Ambion). $1 \mu \mathrm{g}$ of total RNA was used for cDNA synthesis with a PrimeScriptTM RT reagent kit (Takara) according to the instruction provided by the manufacturer. qPCR was carried out using SYBR premix EX Taq (TaKaRa) and ROX Reference Dye (ROX) and analyzed with Stratagene Mx3000p (Agilent Technologies).

Western blot analysis. Western blotting was performed as described before [23]. Briefly, cells were harvested, boiled in SDS loading buffer and resolved on SDS-PAGE.

Chromatin immunoprecipitation (ChIP) assay. A549 cells were first cross-linked with $1 \%$ formaldehyde for $10 \mathrm{~min}$ at room temperature. Glycine $(0.125 \mathrm{M})$ was added to stop the reaction. Treated cells were suspended in lysis buffer $(50 \mathrm{mM}$ Tris-HCl, pH 8.1, 1\% SDS, 10 mM EDTA and protease inhibitors). Protein A/G-Sepharose beads conjugated with either anti-GFP antibody or control IgG were used for incubation with cell lysates. After elution, the resulting reactants were amplified by PCR.

RNA immunoprecipitation. RIP was performed as described previously [24]. Briefly, $1 \sim 3 \times 10^{7}$ cells were lysed in hypotonic buffer supplemented with RNase A inhibitor and DNase I before centrifugation. Cell lysates were pre-cleared with protein $\mathrm{A} / \mathrm{G}$ beads (Pierce) before they were incubated with protein $\mathrm{A} / \mathrm{G}$ beads coated with the indicated antibodies at $4^{\circ} \mathrm{C}$ for $3 \mathrm{~h}$. After extensive washing, the bead-bound immunocomplexes were eluted using elution buffer $(50 \mathrm{mM}$ Tris, pH 8.0, $1 \%$ SDS, and $10 \mathrm{mM}$ EDTA) at $65^{\circ} \mathrm{C}$ for $10 \mathrm{~min}$. To isolate protein associated RNAs from the eluted immunocomplexes, samples were treated with proteinase $\mathrm{K}$ and RNAs were extracted by phenol/chloroform. Purified RNAs were then subjected to RT-PCR analysis.

Colony formation assay. A549 cells transfected with scramble, GATA2 or GATA2-AS1 siRNAs were plated at a density of 500 cells per well on a six-well plate. After one week, cells were then fixed with $10 \%$ cold methanol for $5 \mathrm{~min}$ and stained with $0.005 \%(\mathrm{~m} / \mathrm{v})$ crystal violet for $30 \mathrm{~min}$ at room temperature. After extensive wash, the colonies were photographed.

Cell cycle analysis. Cells were harvested, washed and fixed with $70 \%(\mathrm{v} / \mathrm{v})$ ethanol at $4^{\circ} \mathrm{C}$ overnight. After fixation, cells were centrifuged for $5 \mathrm{~min}$ at $1200 \times \mathrm{g}$, washed twice with PBS and incubated in PBS containing $40 \mathrm{mg} / \mathrm{ml}$ propidium iodide (Sigma), $200 \mathrm{mg} / \mathrm{ml} \mathrm{RNase} \mathrm{A} \mathrm{(Sigma)} \mathrm{for} 30 \mathrm{~min}$ at room temperature. Cell cycle distribution was examined by flow cytometry using a FACScan flow cytometer (Becton Dickinson, Oxford, UK).

Reproducibility. All the data were repeated at least three times. The western blot analyses were representative of three independent experiments.

Statistical analysis. Analysis was performed using Microsoft Excel and GraphPad Prism to assess differences between varying groups. Data were shown as the mean \pm s.d. from 3 independent experiments. Statistical significance was defined by Student's t test and expressed as a p-value. A p-value $<0.05$ was considered to be statistically significant. One asterisk $\left(^{*}\right)$, two asterisks $\left(^{* *}\right)$ and three asterisks $\left({ }^{* *}\right)$ indicate $\mathrm{p}<0.05$, $\mathrm{p}<0.01$, and $\mathrm{p}<0.001$, respectively.

\section{Results}

GATA2-AS1 inhibits GATA2 in NSCLC cells. As GATA2 is discovered to be critical for the viability of NSCLC cells with RAS mutations, we investigated how is GATA2 regulated in lung cancer cells, and found the gene encoding lncRNA GATA2-AS1 located close to GATA2 on chromosome 3 of human genome, suggesting GATA2-AS1 may play a potential role in regulating GATA2 expression (Figure 1A). Thus we knocked down GATA2-AS1 by siRNA in NSCLS cell line A549 (KRAS mutant) and found mRNA level of GATA2 was indeed upregulated after GATA2-AS1 silencing (Figure 1B). In accordance, GATA2 protein level also increased in GATA2-AS1 deficient cells (Figure 1C), indicating that GATA2-AS1 represses GATA2 expression in NSCLC cells. Furthermore, it has been reported that GATA2 drives lung tumorigenesis through proteasome complex, IL-1/NF-kB and Rho signaling, so we next examined GATA2 targets expression in those three pathways and we found a 
higher expression level in GATA2-AS1 knock down cells (Figure 1D). We also analyzed NF-kB pathway activation and found phosphorylated p65 at ser 276 was indeed enhanced after GATA2-AS1 silencing (Figure 1E). To further investigate whether GATA2-AS1 is able to affect lung cancer cells growth through regulating GATA2, we analyzed A549 cells proliferation with or without silencing of GATA2-AS1 by siRNA. GATA2-AS1 enhanced anchorage-dependent prolif- eration of A549 cells (Figure 1F) and drove those tumor cells from $\mathrm{G} 1$ phase into $S$ phase (Figure $1 \mathrm{G}$ ), demonstrating that GATA2-AS1 represses GATA2 expression and NSCLC cells growth.

GATA2-AS1 binds to GATA1. To understand the mechanism by which GATA2-AS1 inhibits GATA2 expression, we next analyzed GATA2-AS1 subcellular localization, and discovered that it was predominantly located to the nucleus
A

Human chromosome 3

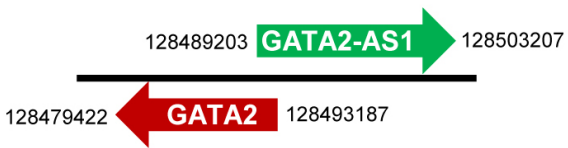

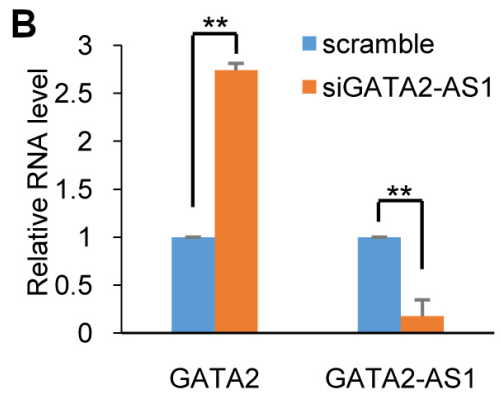
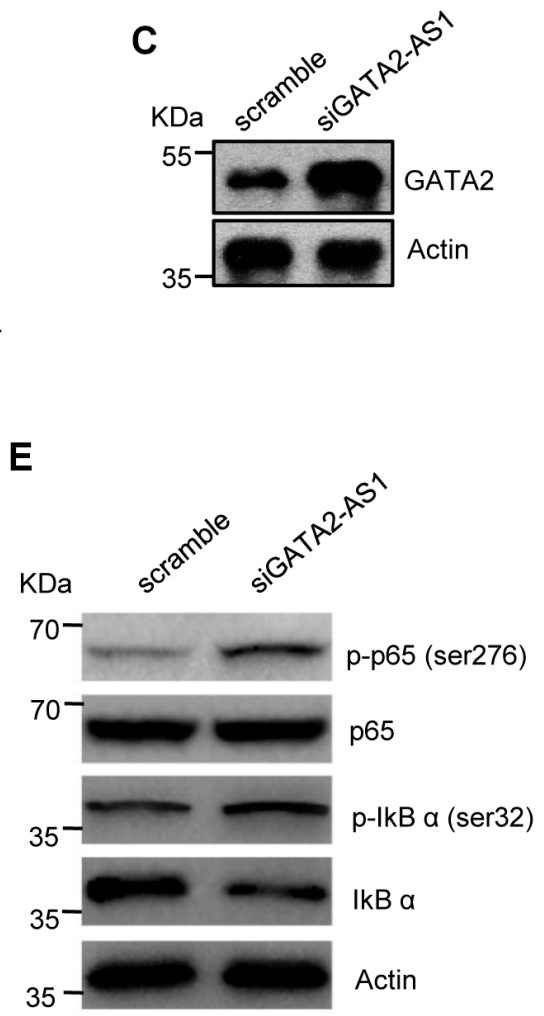

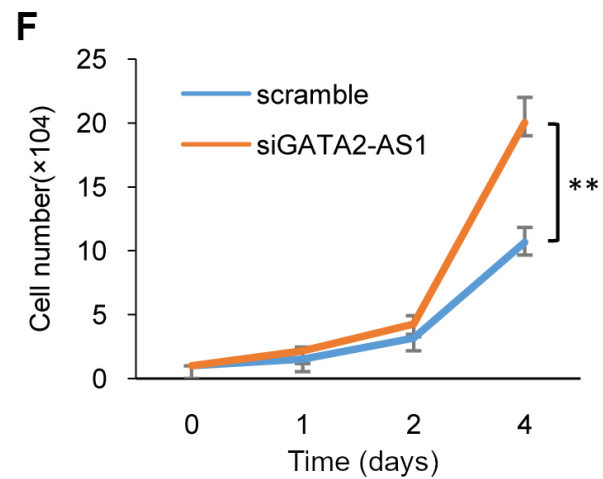

G
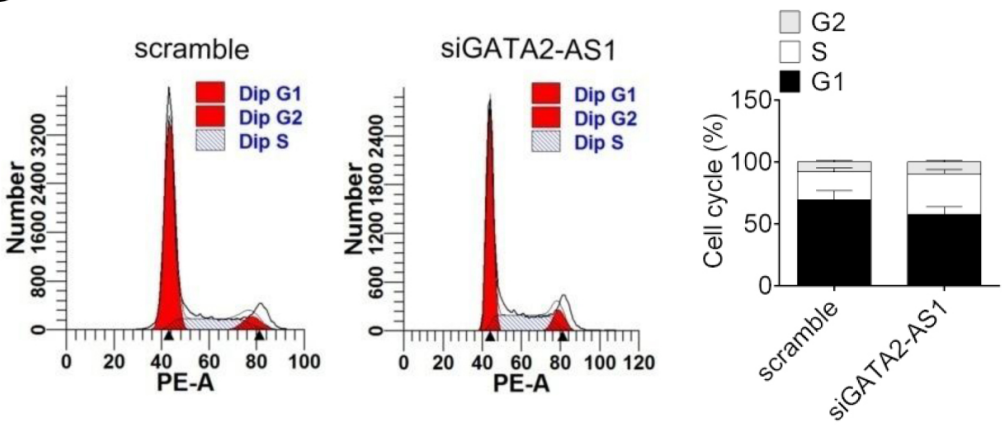

Figure 1. GATA2-AS1 regulates GATA2 in NSCLC cells. A schematic illustration of GATA2-AS1 on human chromosome 3 is shown (A). A549 cells were transfected with GATA2-AS1 or scramble siRNA. Three days later, cells were harvested and mRNA level of GATA2 and GATA2-AS1 (B), amount of GATA protein (C), mRNA level of GATA2 targets (D), protein phosphorylation (E), and cell proliferation (F) were analyzed. A549 cells were transfected with indicated siRNAs and cell cycle analysis was performed $(G)$. Representative results or mean \pm s.d. from 3 independent experiments are shown. ${ }^{*}$ means $\mathrm{p}<0.05,{ }^{* *}$ means $\mathrm{p}<0.01$ and ${ }^{* * *}$ means $\mathrm{p}<0.005$. 
as shown in A549 cells (Figure 2A), which lead us to speculate that GATA2-AS1 may play the role of transcriptional regulator of GATA2 in cis. As GATA1 was reported to bind to GATA2 promoter and repress its transcription, we performed RNA immunoprecipitation (RIP) assays to demonstrate that ectopically expressed GATA1 was able to interact with GATA2-AS1 (Figure 2B). Moreover, we also examined that endogenous GATA2-AS1 was capable of co-precipitating with GATA1 (Figure 2C), indicating GATA2-AS1 and GATA1 protein physically interact with each other in the nucleus. For the co-localization with GATA1 at GATA2 promoter, we further analyzed whether is GATA family co-regulator FOG1 included in the GATA2-AS1 and GATA1 complex using a two-step RIP assay. In the first phase IP we used anti-Flag IgG, which captured high levels of FOG1 and GATA2-AS1 with Flag-GATA1, and in the second IP we used anti-FOG1 IgG co-precipitated Flag-GATA1 and GATA2-AS1 in addition to FOG1 (Figure 2D). These results indicate that GATA1, FOG1 and GATA2-AS1 form a trimer at GATA2 promoter and then regulate its transcription.

GATA2-AS1 enhances repressive function of GATA1. To further study the role of GATA2-AS1 after binding to GATA1 and FOG1, we carried out chromatin immunoprecipitation (ChIP) assay using GATA1 antibody. A lower GATA1 occupancy on GATA2 promoter region, which contains GATA motifs and locates at about $2.8 \mathrm{~kb}$ upstream of GATA2 gene, was detected in GATA2-AS1 silenced cells (Figure 3A), which suggested that GATA2-AS1 increased
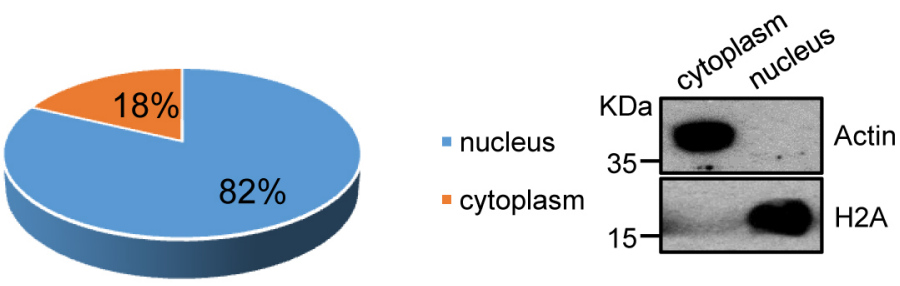
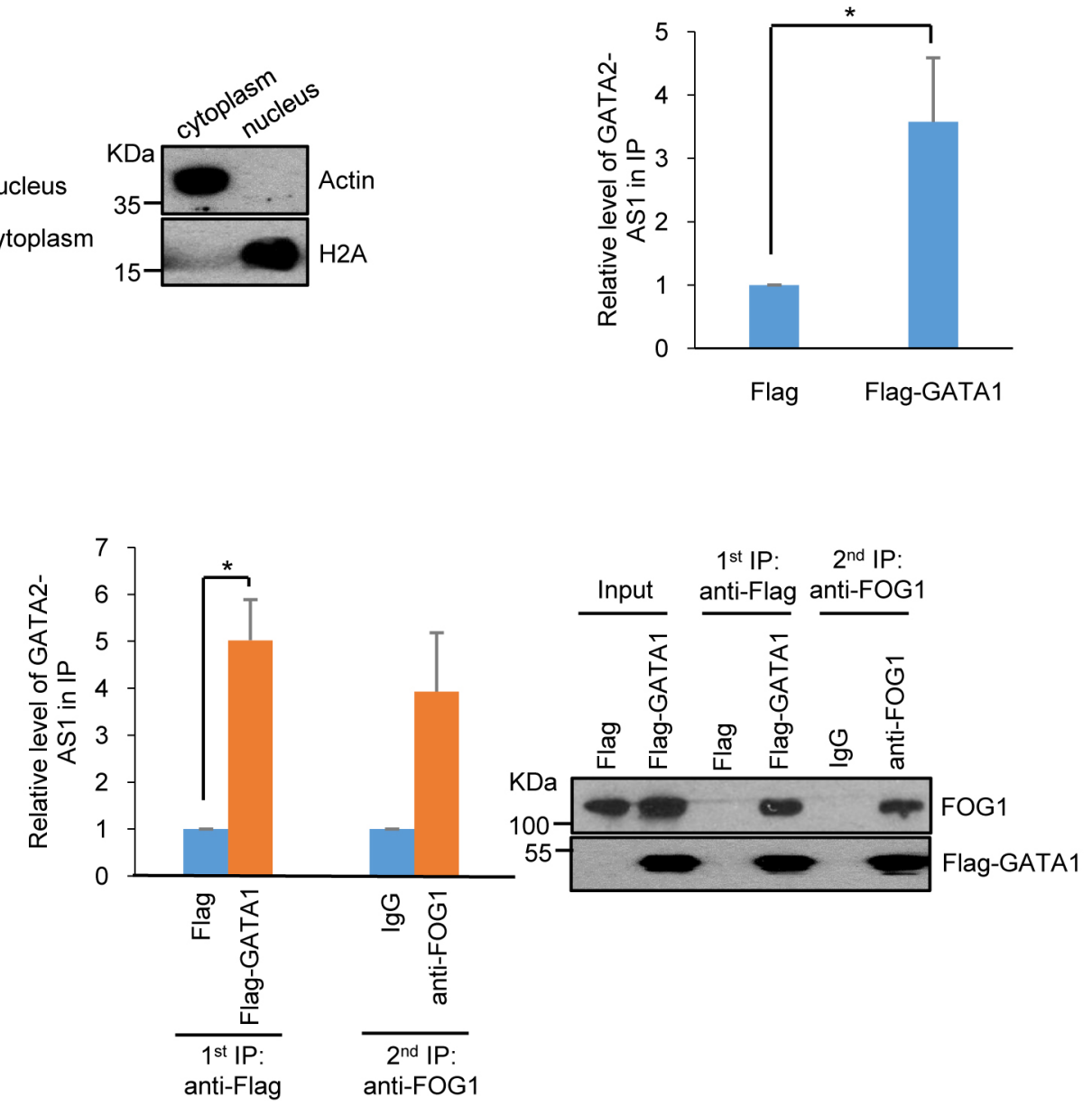

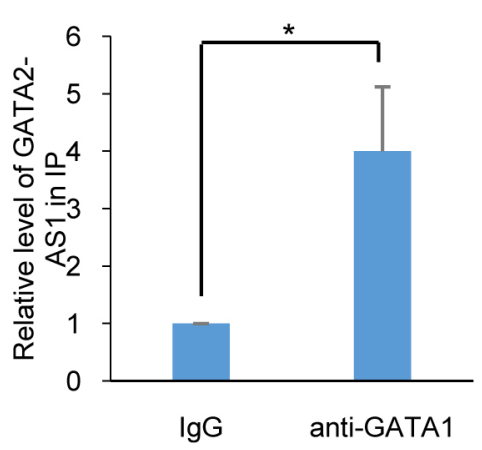

Figure 2. GATA2-AS1 interacts with GATA1. A) Total RNA from cytoplasmic or nuclear fraction of A549 cells was subjected to qPCR analysis of GATA2-AS1 expression and total protein from each fraction was subjected to western blotting analysis. B) A549 cells were transfected with the indicated plasmids. After $48 \mathrm{hr}$ cells were harvested and subjected to RIP with Flag antibody. Total RNAs were extracted followed by qPCR analysis. C) A549 cells were harvested and subjected to RIP with GATA1 antibody or isotype-matched immunoglobulin G (IgG). Total RNAs were extracted and relative level of GATA2-AS1 was analyzed by qPCR. D) Whole cell lysates from A549 cells transduced with Flag-Vector or Flag-GATA1 were subjected to IP using an anti-Flag antibody. The resulting precipitates were then eluted using Flag peptides. Ten percent of the eluents were subjected to western blotting analysis using an anti-Flag antibody. Ten percent of the eluents were subjected to qPCR analysis of GATA2-AS1 expression. Eighty percent of the eluents were subjected to a secondary phase of IP using an anti-FOG1 antibody. Ten percent of the resulting precipitates were subjected to western blotting analysis and the rest of the eluents were subject to qPCR analysis of GATA2-AS1 expression. 

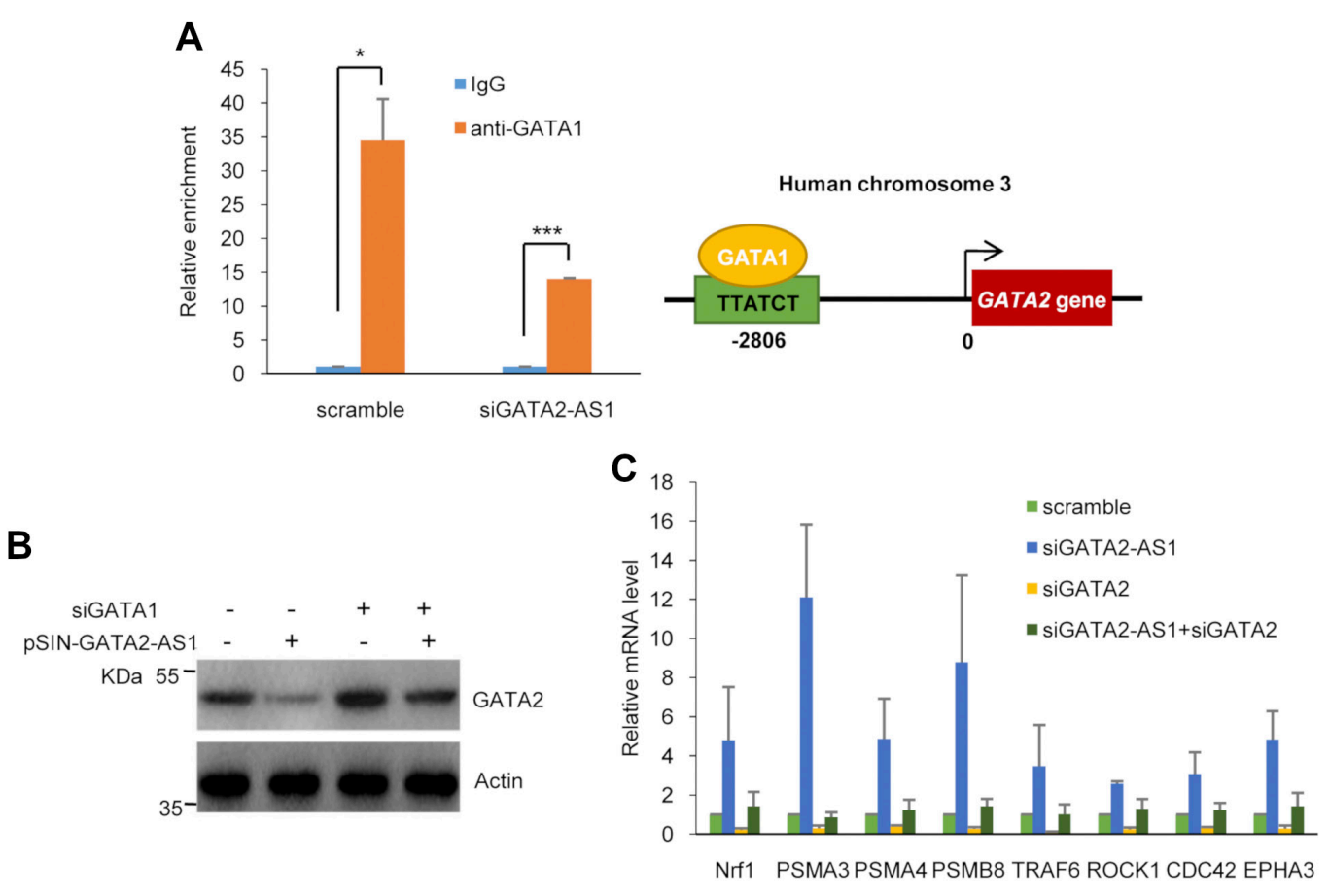

D

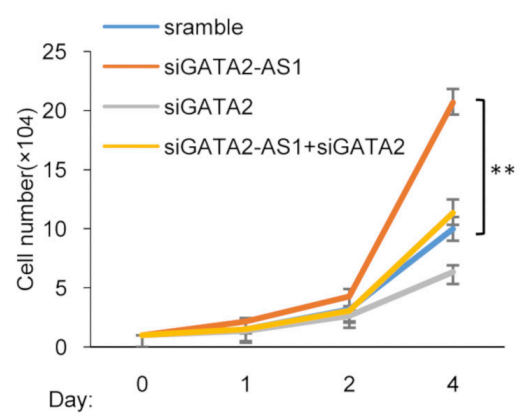

$\mathbf{F}$
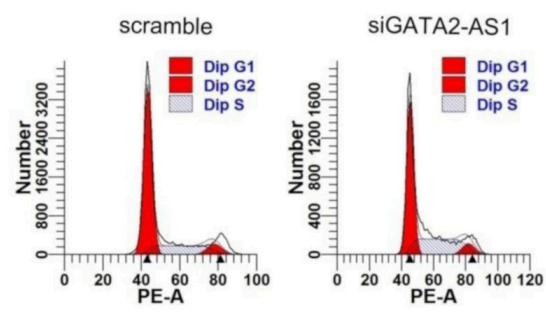

E
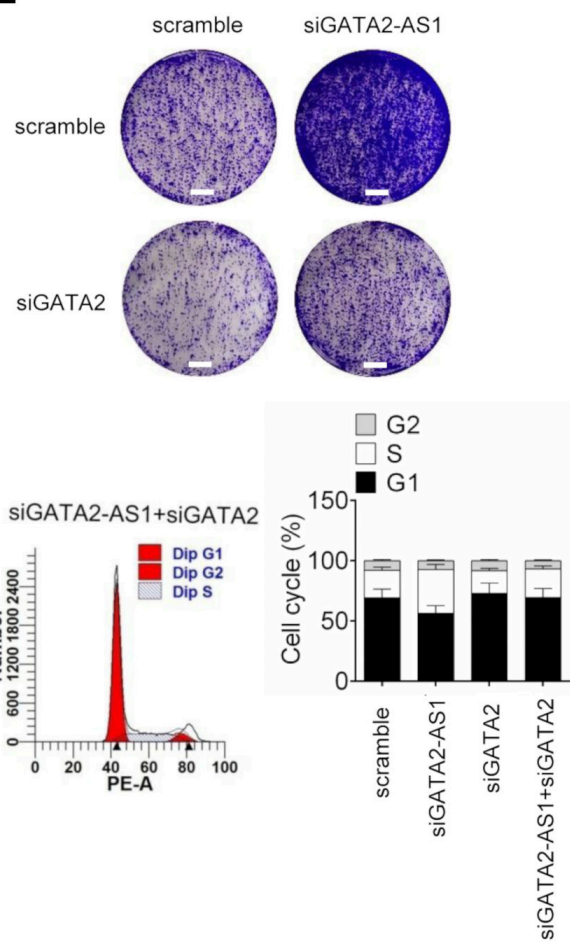

Figure 3. GATA2-AS1 affects tumor cell growth via GATA2. A) Cell lysates of A549 cells transfected with GATA2-AS1 or scramble siRNA were subjected to ChIP assays. ChIP products were analyzed by $\mathrm{QPCR}$ using primers directed to $2.8 \mathrm{~kb}$ upstream of the GATA2 promoter. B) A549 cells were transfected with GATA2-AS1 expression plasmid pSIN-GATA2-AS1 or empty vector and indicated siRNAs. Three days later, cells were harvested and cell lysates were analyzed by western blotting. C) A549 cells were transfected with indicated siRNAs. Three days later, cells were harvested, and mRNA level of GATA2 targets were analyzed by qPCR. D) A549 cells were transfected with indicated siRNAs and cell number was counted at indicated times. E) GATA2-AS1 silencing promoted clonogenicity in A549 cells, which was rescued by GATA2 siRNA. Scale bar: $0.5 \mathrm{~cm}$. F) A549 cells were transfected with indicated siRNAs and cell cycle analysis $\left(1 \times 10^{4}\right.$ cells $)$ were measured by FACS after PI staining, and the percentage of cells in each phase of cell cycle is labeled in the figure. 
GATA1 binding to GATA2 promoter and therefore enhanced the repressive function of GATA1 on GATA2 expression. We knocked down GATA1 concurrently with GATA2-AS1 overexpression, and found that the suppressive effect of GATA2-AS1 on GATA2 expression was rescued by GATA1 silencing, which further confirmed GATA2-AS1 regulation of GATA2 via GATA1 (Figure 3B).

GATA2-AS1 inhibits NSCLC cells proliferation via GATA2. After understanding the mechanism how GATA2AS1 regulates GATA2, we next sought to determine whether GATA2-AS1 indeed inhibited NSCLC cells proliferation via regulating GATA2 expression. We first examined GATA2 targets expression in A549 cells with or without in GATA2AS1 siRNA transfection and found those alterations in GATA2-AS1 silenced cells could be reversed by GATA2 siRNA treatment (Figure 3C), suggesting that GATA2 indeed mediates the effect of GATA2-AS1 on NSCLC cells growth. Moreover, in anchorage-dependent tumor cell proliferation assays we demonstrated that the phenotype of GATA2AS1 silenced A549 cells could be rescued by GATA2 siRNA (Figure $3 \mathrm{D}$ and $\mathrm{E}$ ), which was further confirmed by cell cycle analysis between GATA2 knock down and GATA2-AS1 and GATA2 double knock down cells (Figure 3F), illustrating the mechanism GATA2-AS1 regulates lung cancer growth through GATA2.

MYC transcriptionally regulates GATA2-AS1. Our finding that the level of GATA2-AS1 is closely correlated with NSCLC cells proliferation lead us to further test the possibility that GATA2-AS1 expression is also strictly regulated during tumorigenesis. Through bioinformatic analyses, a MYC response element sequence (CACGTG) was determined at the -1964 site of GATA2-AS1 promoter (Figure 4A). Remarkably, MYC silencing induced upregulation of GATA2-AS1 level in human NSCLC cells (Figure 4B), and in contrast, when increasing exogenous MYC were transfected into A549 cells, GATA2-AS1 expression levels were gradually reduced (Figure 4C). To identify which region of the GATA2AS1 promoter is bound and thus repressed by MYC, we employed an anti-MYC antibody and performed ChIP assay. We found MYC indeed bound to the region including the MYC response element at -1964 of the GATA2-AS1 promoter (Figure 4D). Furthermore, as Miz1 plays important role in

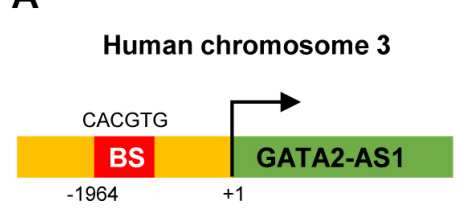

B
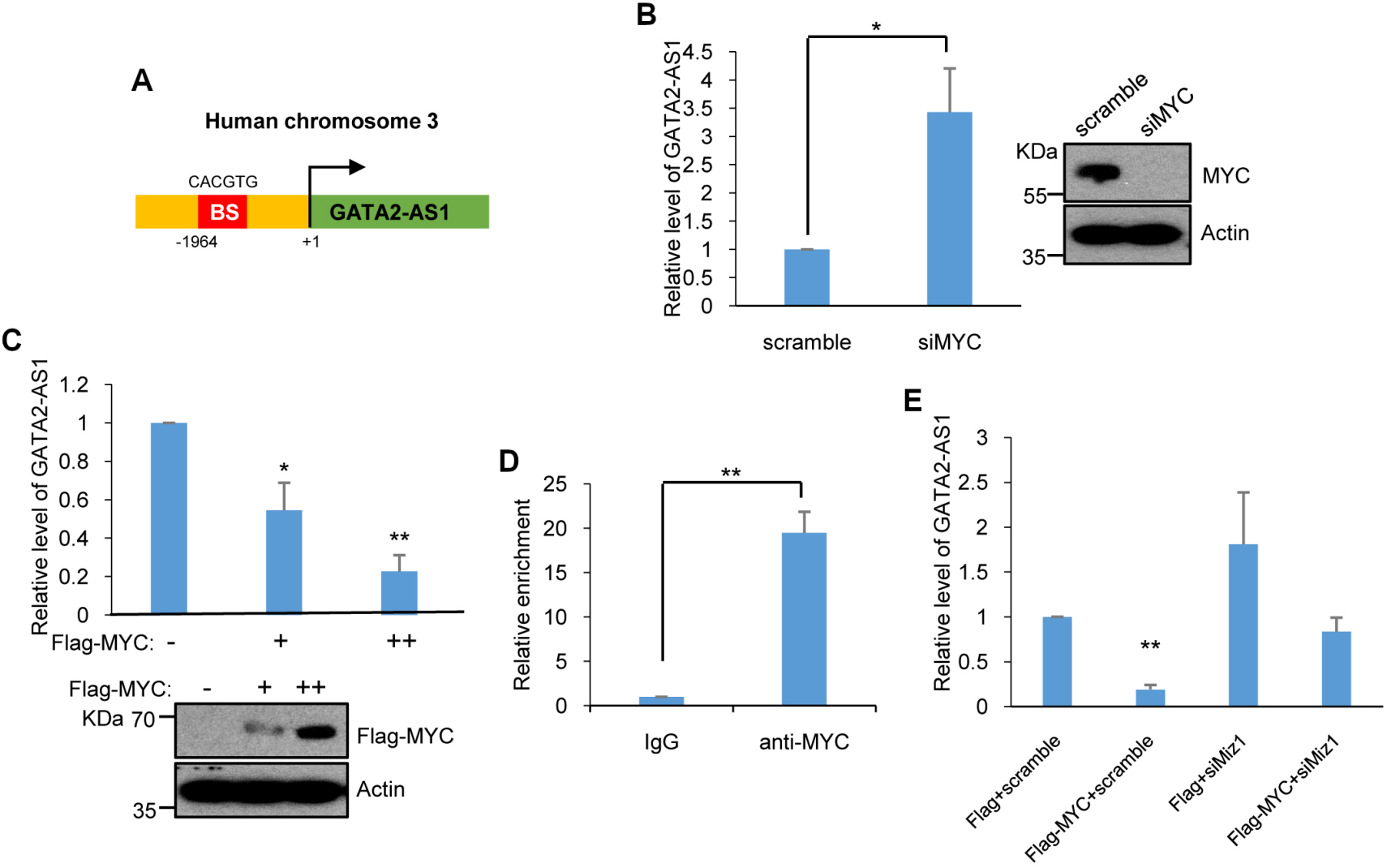

Figure 4. GATA2-AS1 is transcriptionally inhibited by MYC. A) Schematic illustration of MYC binding site at GATA2-AS1 promoter. B) A549 cells were transfected with MYC or scramble siRNAs. Three days later, cells were harvested and expression level of GATA2-AS1 was analyzed by qPCR. C) A549 cells were transfected with Flag-vector or Flag-tagged MYC at increasing concentrations, and total RNA and cell lysates were subjected to qPCR and western blotting analysis respectively. D) Cell lysates of A549 cells transfected with MYC or scramble siRNAs were subjected to ChIP assays. E) A549 cells were transfected with plasmids and siRNAs. Three days later, cells were harvested and expression level of GATA2-AS1 was analyzed by qPCR. 
MYC-mediated transcriptional repression of gene expression [25], we next knocked down Mizl expression by siRNA silencing and found Mizl deficiency was able to rescue the GATA2-AS1 downregulation induced by MYC overexpression (Figure 4E), indicating MYC regulates transcriptional repression of GATA2-AS1 via interacting with Miz1.

GATA2-AS1 is expressed at a lower level in cancer tissues. To further understand the function of GATA2-AS1 during lung cancer development, we examined GATA2AS1 expression in clinical NSCLC samples. Analysis of surgically removed non-small cell lung cancer samples showed that most of cancer tissues expressed lower levels of GATA2-AS1 compared with adjacent noncancerous tissues (Figure 5A). Consistently, these lung cancer tissues also showed a relatively higher MYC and GATA2 expression (Figure 5B). However, there was no significant difference between the level of GATA1 in cancer tissues and normal tissues (Figure 5B). These results suggest that GATA2-AS1 plays a tumor suppressive role through inhibition of cancer cell proliferation.
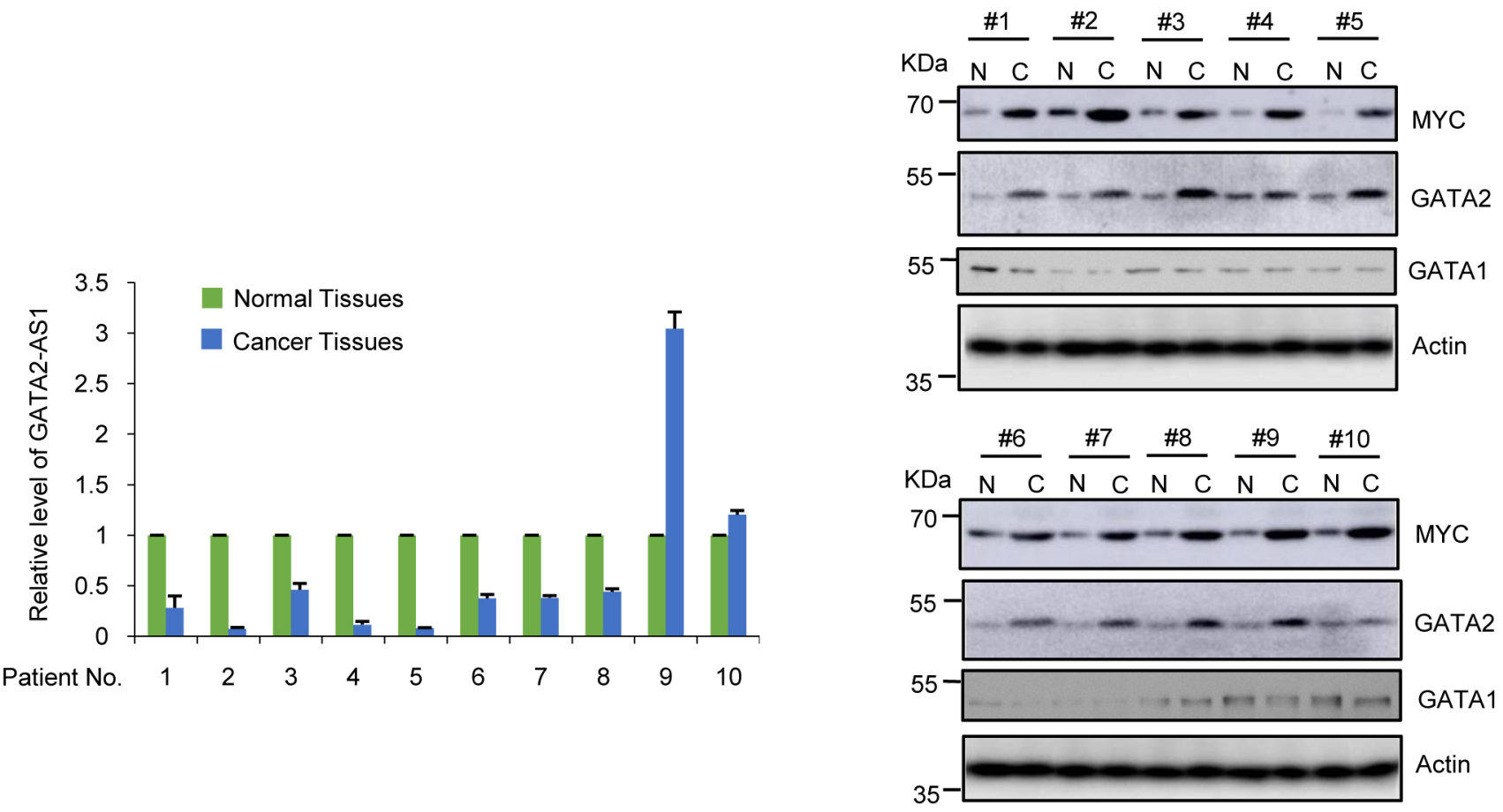

Figure 5. GATA2-AS1 expression in clinical samples. A) Total RNA of samples from cancer or adjacent normal tissues of NSCLC patients were extracted and analyzed by qPCR. B) Samples from cancer or adjacent normal tissues of NSCLC patients were analyzed by western blotting.

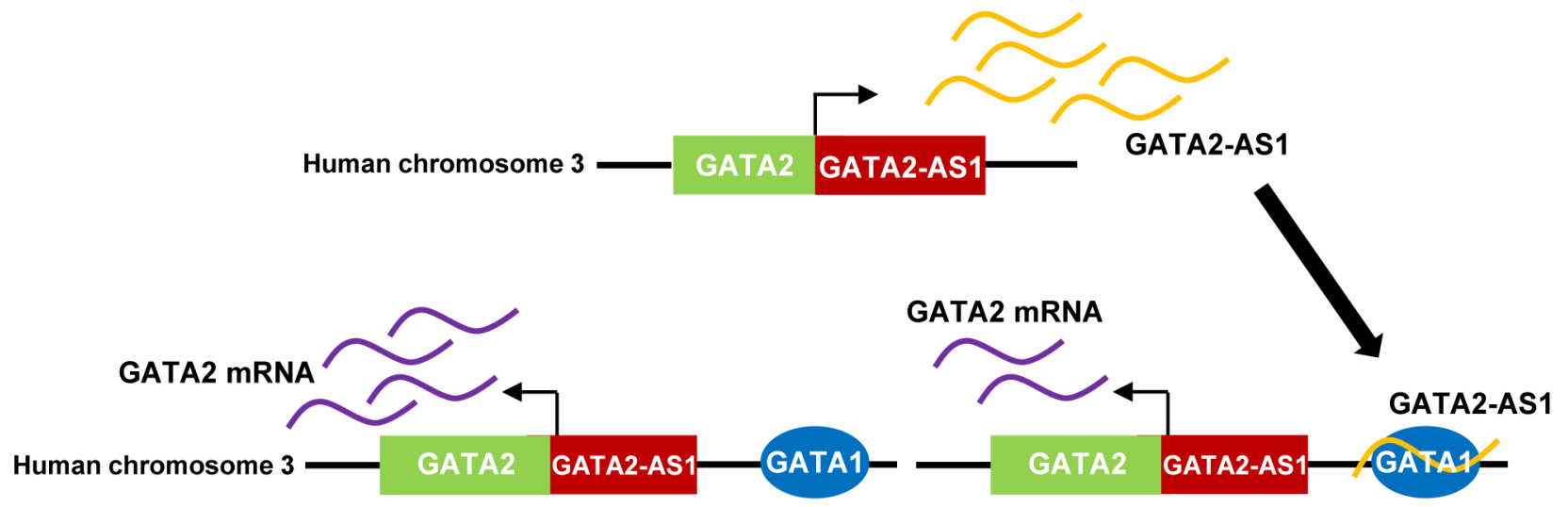

Figure 6. A propose model illustrating GATA2-AS1-GATA1-GATA2 axis in NSCLC cells. 


\section{Discussion}

In this study we discovered a lncRNA GATA2-AS1 is involved in non-small cell lung cancer growth and development, which accounts for $85 \%$ of all types of lung cancer histologically in clinic [1]. We found that GATA2-AS1 suppressed tumor cell proliferation via regulating GATA2 expression. According to previous studies, GATA2 is a driving force of development of NSCLC with RAS pathway mutations, which maintains NSCLC cells survival via modulation of three pathways: proteasome complex, IL-1/NF-kB signaling and Rho-signaling [4]. Here we showed that GATA2 and these three downstream pathways indeed mediated GATA2AS1 functions, illustrating how GATA2 is regulated during tumorigenesis of non-small cell lung cancer.

GATA2-AS1 is located at antisense strand of GATA2 gene on human chromosome 3, and after being transcribed, GATA2-AS1 plays its regulatory role in cis. We analyzed GATA2-AS1 subcellular localization and postulated that it interacted in nucleus with GATA1 protein, which is a negative regulator on GATA2 expression and facilitated GATA1 to bind to GATA2 promoter. However, we noticed that GATA2-AS1 and GATA2 double knockdown didn't fully rescued the acceleration of tumor cell growth with GATA2-AS1 deficiency, suggesting that there are also other downstream pathways mediated GATA2-AS1 function in NSCLC cells and it needs further investigation.

MYC expression is upregulated in many types of cancers including NSCLC $[10,26,27]$ and in our study we found that GATA2-AS1 is inhibited by MYC in NSCLC cells. We demonstrated that MYC bound at promoter region of GATA2-AS1 and repressed GATA2-AS1 expression through interacting with Miz1. As a result, the level of GATA2-AS1 will be lower in tumor comparing with normal cells, and its repressive effect on GATA2 will be partially relieved in NSCLC, which may lead to tumor growth and reveals an unknown mechanism that MYC may exert its positive function in tumorigenesis.

Furthermore, we examined GATA2-AS1 expression in clinical NSCLC samples and found in majority of the samples the level of GATA2-AS1 was relatively lower in cancer tissues. Consistently, GATA2 and MYC expression was higher in cancer tissues in these samples. However, it is also observed that in few samples GATA2-AS1 level was higher in cancer tissues than in adjacent normal ones. We tend to attribute this phenotype to the fact that they are not RAS pathway mutations samples as GATA2 only induces cancer cell proliferation in NSCLC with RAS pathway mutations, which need further investigation. We also found that the expressions of GATA1 in cancer tissues and normal tissues were at the same levels. As we postulated that GATA2-AS1 affected transcriptional activity rather than expression level of GATA1, and we believe that this phenotype is consistent with our previous findings.

Taken together, our findings elaborate the MYC-GATA2AS1-GATA2 pathway in NSCLC development and provide potential targets for lung cancer treatment.
Supplementary information is available in the online version of the paper.

Acknowledgements: This work was supported by grants from the National Natural Science Foundation of China (21304028).

\section{References}

[1] FERLAY J, SHIN HR, BRAY F, FORMAN D, MATHERS C et al. Estimates of worldwide burden of cancer in 2008: GLOBOCAN 2008. Int J Cancer 2010; 127: 2893-2917. https:// doi.org/10.1002/ijc. 25516

[2] ENGELMAN JA, CHEN L, TAN X, CROSBY K, GUIMARAES AR et al. Effective use of PI3K and MEK inhibitors to treat mutant Kras G12D and PIK3CA H1047R murine lung cancers. Nat Med 2008; 14: 1351-1356. https://doi. org/10.1038/nm.1890

[3] LUO J, EMANUELE MJ, LI D, CREIGHTON CJ, SCHLA$\mathrm{BACH}$ MR et al. A genome-wide RNAi screen identifies multiple synthetic lethal interactions with the Ras oncogene. Cell 2009; 137: 835-848. https://doi.org/10.1016/j. cell.2009.05.006

[4] KUMAR MS, HANCOCK DC, MOLINA-ARCAS M, STECKEL M, EAST $P$ et al. The GATA2 transcriptional network is requisite for RAS oncogene-driven non-small cell lung cancer. Cell 2012; 149: 642-655. https://doi. org/10.1016/j.cell.2012.02.059

[5] PATIENT RK, MCGHEE JD. The GATA family (vertebrates and invertebrates). Curr Opin Genet Dev 2002; 12: 416-422. https://doi.org/10.1016/S0959-437X(02)00319-2

[6] GRASS JA, BOYER ME, PAL S, WU J, WEISS MJ et al. GATA-1-dependent transcriptional repression of GATA-2 via disruption of positive autoregulation and domain-wide chromatin remodeling. Proc Natl Acad Sci U S A 2003; 100: 8811-8816. https://doi.org/10.1073/pnas. 1432147100

[7] MARTOWICZ ML, GRASS JA, BOYER ME, GUEND H, BRESNICK EH. Dynamic GATA factor interplay at a multicomponent regulatory region of the GATA-2 locus. J Biol Chem 2005; 280: 1724-1732. https://doi.org/10.1074/jbc. M406038200

[8] VARMUS HE. The molecular genetics of cellular oncogenes. Annu Rev Genet 1984; 18: 553-612. https://doi.org/10.1146/ annurev.ge.18.120184.003005

[9] BLACKWOOD EM, EISENMAN RN. Max: a helix-loophelix zipper protein that forms a sequence-specific DNAbinding complex with Myc. Science 1991; 251: 1211-1217. https://doi.org/10.1126/science.2006410

[10] MEYER N, PENN LZ. Reflecting on 25 years with MYC. Nat Rev Cancer 2008; 8: 976-990. https://doi.org/10.1038/ nrc2231

[11] ARMELIN HA, ARMELIN MC, KELLY K, STEWART T, LEDER $\mathrm{P}$ et al. Functional role for c-myc in mitogenic response to platelet-derived growth factor. Nature 1984; 310: 655-660. https://doi.org/10.1038/310655a0

[12] DANG CV, MCGUIRE M, BUCKMIRE M, LEE WM. Involvement of the 'leucine zipper' region in the oligomerization and transforming activity of human c-myc protein. Nature 1989; 337: 664-666. https://doi.org/10.1038/337664a0 
[13] STEFFEN D. Proviruses are adjacent to c-myc in some murine leukemia virus-induced lymphomas. Proc Natl Acad Sci U S A 1984; 81: 2097-2101. https://doi.org/10.1073/ pnas.81.7.2097

[14] CREWSS, BARTHR,HOODL,PREHNJ,CALAMEK.Mouse c-myc oncogene is located on chromosome 15 and translocated to chromosome 12 in plasmacytomas. Science 1982; 218: 1319-1321. https://doi.org/10.1126/science.7146913

[15] COLLINS S, GROUDINE M. Amplification of endogenous myc-related DNA sequences in a human myeloid leukaemia cell line. Nature 1982; 298: 679-681. https://doi. org/10.1038/298679a0

[16] PANZITT K, TSCHERNATSCH MM, GUELLY C, MOUSTAFA T, STRADNER $M$ et al. Characterization of HULC, a novel gene with striking up-regulation in hepatocellular carcinoma, as noncoding RNA. Gastroenterology 2007; 132: 330-342. https://doi.org/10.1053/j.gastro.2006.08.026

[17] TAKAHASHI K, YAN I, HAGA H, PATEL T. Long noncoding RNA in liver diseases. Hepatology 2014; 60: 744-753. https://doi.org/10.1002/hep. 27043

[18] MORRIS KV, MATTICK JS. The rise of regulatory RNA. Nat Rev Genet 2014; 15: 423-437. https://doi.org/10.1038/ $\operatorname{nrg} 3722$

[19] ZHAO J, SUN BK, ERWIN JA, SONG JJ, LEE JT. Polycomb proteins targeted by a short repeat RNA to the mouse X chromosome. Science 2008; 322: 750-756. https://doi. org/10.1126/science.1163045

[20] WANG KC, CHANG HY. Molecular mechanisms of long noncoding RNAs. Mol Cell 2011; 43: 904-914. https://doi. org/10.1016/j.molcel.2011.08.018
[21] WANG X, ARAI S, SONG X, REICHART D, DU K et al. Induced ncRNAs allosterically modify RNA-binding proteins in cis to inhibit transcription. Nature 2008; 454: 126-130. https://doi.org/10.1038/nature06992

[22] DEAN NM, MCKAY R, CONDON TP, BENNETT CF. Inhibition of protein kinase $\mathrm{C}$-alpha expression in human A549 cells by antisense oligonucleotides inhibits induction of intercellular adhesion molecule 1 (ICAM-1) mRNA by phorbol esters. J Biol Chem 1994; 269: 16416-16424.

[23] ESTEBAN JI, SHIH JW, TAI CC, BODNER AJ, KAY JW et al. Importance of Western blot analysis in predicting infectivity of anti-HTLV-III/LAV positive blood. Lancet 1985; 2: 1083-1086. https://doi.org/10.1016/S0140-6736(85)90683-X

[24] MCHUGH CA, CHEN CK, CHOW A, SURKA CF, TRAN $\mathrm{C}$ et al. The Xist lncRNA interacts directly with SHARP to silence transcription through HDAC3. Nature 2015; 521: 232-236. https://doi.org/10.1038/nature14443

[25] STALLER P, PEUKERT K, KIERMAIER A, SEOANE J, LUKAS J et al. Repression of p15 INK4b expression by Myc through association with Miz-1. Nat Cell Biol 2001; 3: 392 399. https://doi.org/10.1038/35070076

[26] HSIEH AL, WALTON ZE, ALTMAN BJ, STINE ZE, DANG CV. MYC and metabolism on the path to cancer. Semin Cell Dev Biol 2015; 43: 11-21. https://doi.org/10.1016/j. semcdb.2015.08.003

[27] RAPP UR, KORN C, CETECI F, KARREMAN C, LUETKENHAUS $\mathrm{K}$ et al. MYC is a metastasis gene for nonsmall-cell lung cancer. PLoS One 2009; 4: e6029. http://doi. org/10.1371/journal.pone.0006029 